Volume 10, No.3, May - June 2021

International Journal of Advanced Trends in Computer Science and Engineering

Available Online at http://www.warse.org/IJATCSE/static/pdf/file/ijatcse241032021.pdf

https://doi.org/10.30534/ijatcse/2021/241032021

\title{
Deriving Heart Rate and Respiratory Rate from Pulse Oximetry Data
}

\author{
Jennifer C. Dela Cruz ${ }^{1}$, John Paul D. Alolor ${ }^{2}$, Rouella Christine L. Panganiban ${ }^{3}$, \\ Rhianna Marielle G. Victorio ${ }^{4}$, Jonathan Ibera ${ }^{5}$ \\ ${ }^{1}$ Mapúa University, Philippines, jcdelacruz@ mapua.edu.ph \\ ${ }^{2}$ Mapúa University, Philippines, jpdalolor@mymail.mapua.edu.ph \\ ${ }^{3}$ Mapúa University, Philippines, rclpanganiban@mymail.mapua.edu.ph \\ ${ }^{4}$ Mapúa University, Philippines, rmgvictorio@ mymail.mapua.edu.ph \\ ${ }^{5}$ Cal Poly Pomona, United States, jonibera@yahoo.com
}

\begin{abstract}
Respiratory Rate (RR) is a vital sign representing air movement in and out of the body. Neglecting the respiratory rate can foresee the patient being at risk. The lack of portable device in a point of care unit, i.e. ambulances, can be a demanding task. In this study, the researchers developed a hardware device that can measure resting Heart Rate (HR) and RR from a custom pulse oximeter tethered to the Arduino board using signal processing to extract HR and Symlet Wavelet Transform to extract the RR. The derived HR and RR from a custom pulse oximeter is compared to a clinical-grade pulse oximeter and yielded an MSE of around 3.8. Moreover, results obtained from the custom pulse oximeter vs the reference are close and valid.
\end{abstract}

Key words: Fast Fourier Transform, Heart Rate, Respiratory Rate, Symlet Wavelet Transform

\section{INTRODUCTION}

Respiratory rate is a vital sign representing air movement in and out of the body or the number of breaths per minute. Typically, the range of the respiratory rate of individuals varies. Among the vital signs, the respiratory rate is usually excluded or inaccurately measured. Furthermore, respiratory rate change can see early signs of patient deterioration since oxygen is being carried out to other tissues while being maintained [1]. Neglecting the respiratory rate can foresee the patient being at risk. Although devices can measure the respiratory rate (ex. Impedance pneumography and capnography monitors), it is very costly and is only available or accessible in sensitive or critical areas [2]. Developing a hardware device that can measure the resting heart rate through photoplethysmography signals that will extract and derive the respiratory rate using different Digital Signal Processing algorithms that can maximize the use of pulse oximeters that are cheap, readily available, and can use at any point of care.

The Photoplethysmogram is a device used to detect blood volume changes in the microvascular bed of tissue. The pulse oximeter will be used to obtain photoplethysmogram data, and it can be worn on the fingers. The pulse oximeter only measures the heart or pulse rate, but with the application of a powerful signal processing, the respiration rate information can be extracted. The pulse oximeter will be configured and derived using wavelet transform to get the right algorithm to harness the frequency needed to detect the respiration rate. It has been learned that there is a relationship between the heart rate and respiratory rate. The respiratory variation can affect the heart rate. Also, using spectral analysis of heart rate variability, the best peak shows changes in the interval at the same frequency as respiration [3].

Respiration rate is a crucial vital sign; however, people often neglect it. It can foresee the patient being at risk. Although one of the most important vital signs, the problem is that there is no available portable device that can measure respiratory rate, especially in point of care units such as ambulances. Acquiring respiration rate can be a demanding task without using a custom pulse oximeter that can measure respiratory rate and may cause a delay in giving proper treatment. For many years, respiration monitor belts and spirometers use to assess air movement in and out of the body, followed by creating an algorithm to detect respiratory rate from ECG signals [4]. Most of the time, respiratory rate measurement is done inside an intensive care unit using patient care monitoring units. These methods, although useful, are much more expensive, laborious because of multiple electrode setups needed, and in some cases, some people get skin reactions to electrodes [5].

In diagnosing cardiac diseases and identifying urgent treatments for critical patients, the ECG signal is generally used. Using Wavelet Transform algorithms aids in analyzing ECG signals. This is because ECG waveforms represents the polarization and depolarization of the heart muscles. Therefore, using Wavelet Transform shows a distinct value on the difference in heart muscle based function [6].

Using Photoplethysmography or PPG, which uses infrared light or pulse oximeter, measures a person's heart blood pressure based on the paper. The pulse rate can be determined using a pulse oximeter to observe the amount of blood passing through the dermis[7]. 
In 2017, Masimo announced the marking of a portable respiratory and heart rate measuring device which is said to be the first pulse oximeter to measure respiration rate. The device itself costs 300 dollars compared to a typical pulse oximeter that costs around 12-13 dollars.

This study's main objective is to develop a hardware device that can measure resting heart rate and respiration rate from a custom pulse oximeter signal tethered to the Arduino board. To be able to attain the main objective, the specific goals established are as follows: (a) to acquire Photoplethysmography data from custom pulse oximeter tethered to Arduino board; (b) to use a Digital Signal Processing algorithm that can filter, window, and estimate the resting HR from the Photoplethysmography raw data using Matlab; (c) to use a Digital Signal Processing algorithm that can extract the respiratory rate accurately from the Photoplethysmography raw data using Matlab; (d) to validate the performance of this device against a reference pulse oximeter (clinical) using multiple test subjects with various heart rate/respiration rate ranges.

This research mainly focuses on developing hardware used to measure resting heart rate and respiration rate from a custom pulse oximeter signal tethered to an Arduino board. This study will conduct with at least 30 samples. Ordinary people of different ages (7-75 years old) and genders using Digital Signal Processing algorithm estimate resting heart rate and respiration rate accurately from Photoplethysmography using Matlab. This research will not cover treatment for a diagnosis regarding heart rate and respiratory rate. Other topics or unrelated information about this research will not be discussed and will be disregarded.

\section{METHODOLOGY}

\subsection{Conceptual Framework}

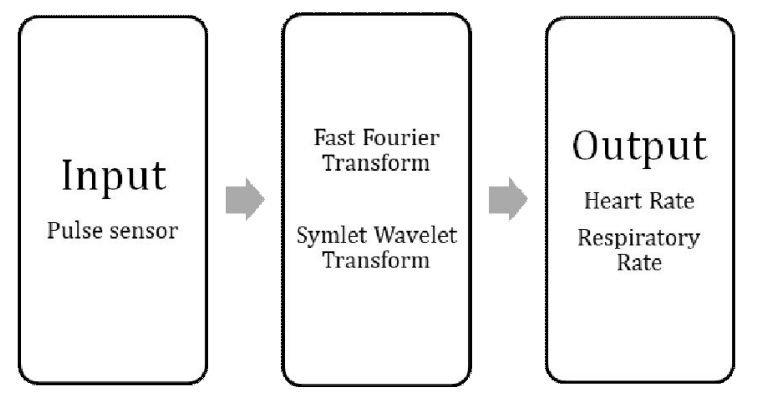

Figure 1: Conceptual Framework

Figure 1 shows the conceptual framework of the study. The input is the pulse sensor from the custom pulse oximeter that receives the signal that contains the photoplethysmography data. A custom pulse oximeter tethered to an Arduino board is programmed to convert the raw photoplethysmography data into a CSV file. The Fast Fourier Transform in MatLab will filter, window, and estimate the photoplethysmography data to get the heart rate from the raw signal. The Symlet Wavelet Transform algorithm will then extract the respiratory rate from the heart rate through MatLab. The outputs shown are the respiratory rate in breaths per minute and the heart rate in beats per minute.

\subsection{Prototype}

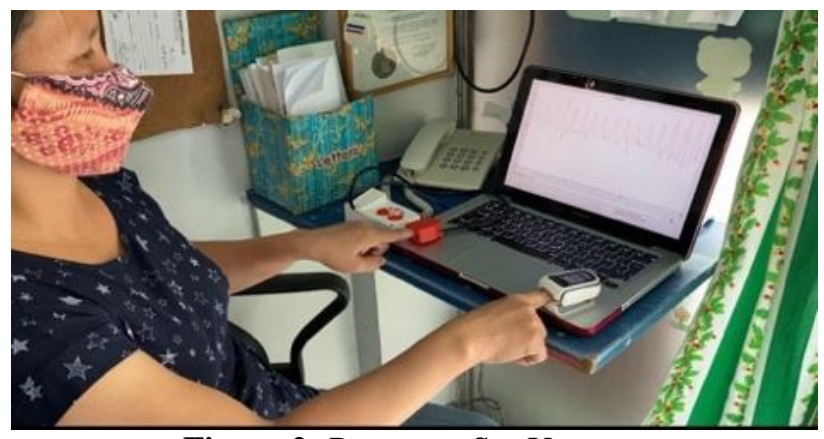

Figure 2: Prototype Set-Up

Figure 2 shows the hardware setup of this study. The monitoring device is directly connected to a laptop for processing and storing of data of the user. Also, connect the monitoring device to a computer before running the graphical user interface. The enclosure of the prototype is 3D Printed and designed to be as portable as possible. The 3D Printed Clipper encloses the pulse sensor that shows compatibility on different age groups. Also, the clipper can be attached or removed from the primary device. It indicates flexibility and comfortability on the user end.

\subsection{Graphical User Interface}

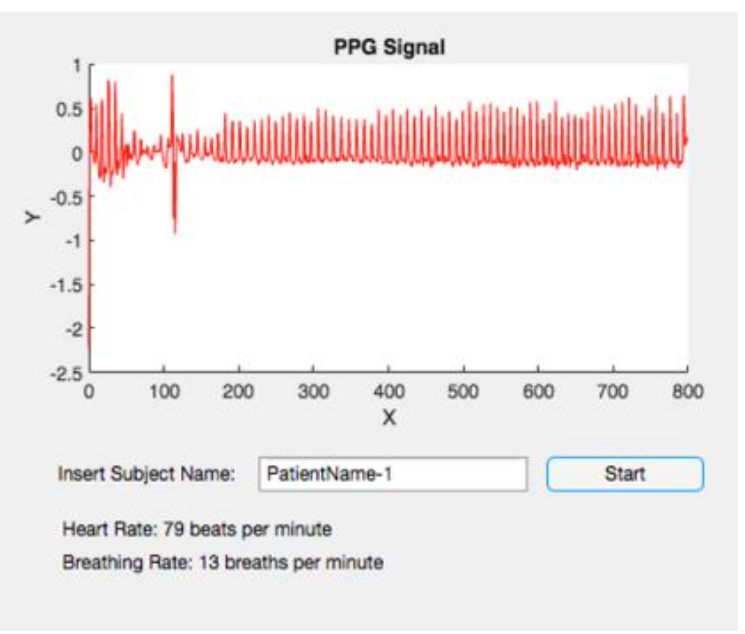

Figure 3: Sample GUI

Figure 3 shows the actual graphical user interface. The graphical user interface can be seen and accessed by running the program. The GUI consists of the PPG signal directly from the sensor. It also includes an input field for the name of the subject or the patient. Press the start button to signal the start of the assessment. Lastly, the two outputs consist of the heart rate measured in beats per minute (bpm) and the breathing rate, which is also the respiratory rate measured in breaths per minute. 


\subsection{Calibration}

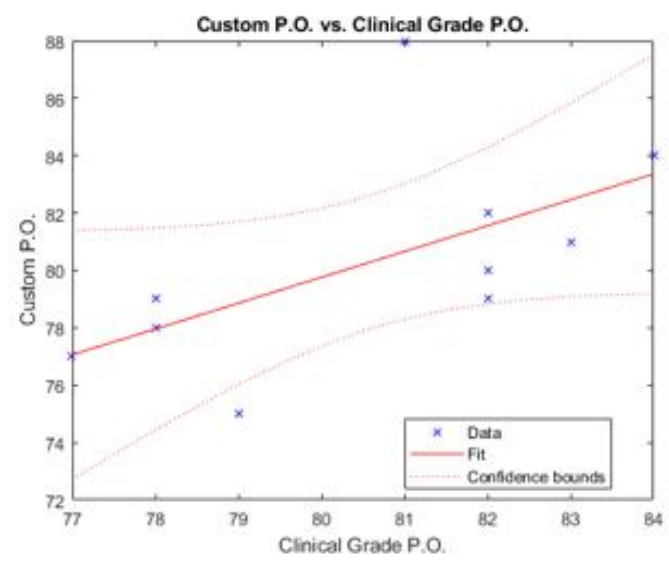

Figure 4: Graph of the Data and Predictions

For the calibration, Matlab is used to process the gathered data. Then, the model created used linear regression. The equation heart_model $=$ fitlm(Custom,Commercial, 1$)$; was used. Lastly, to predict commercial points and custom PPG output given the linear model, the equation ypred $=$ predict(heart_model, Custom); was used. By using these equations, it will acquire the following equation and graph as seen in Figure 4.

Heart Rate Equation: $\mathrm{Y}=0.9008 \mathrm{x}+7.6985$

\subsection{Complete System Flowchart}

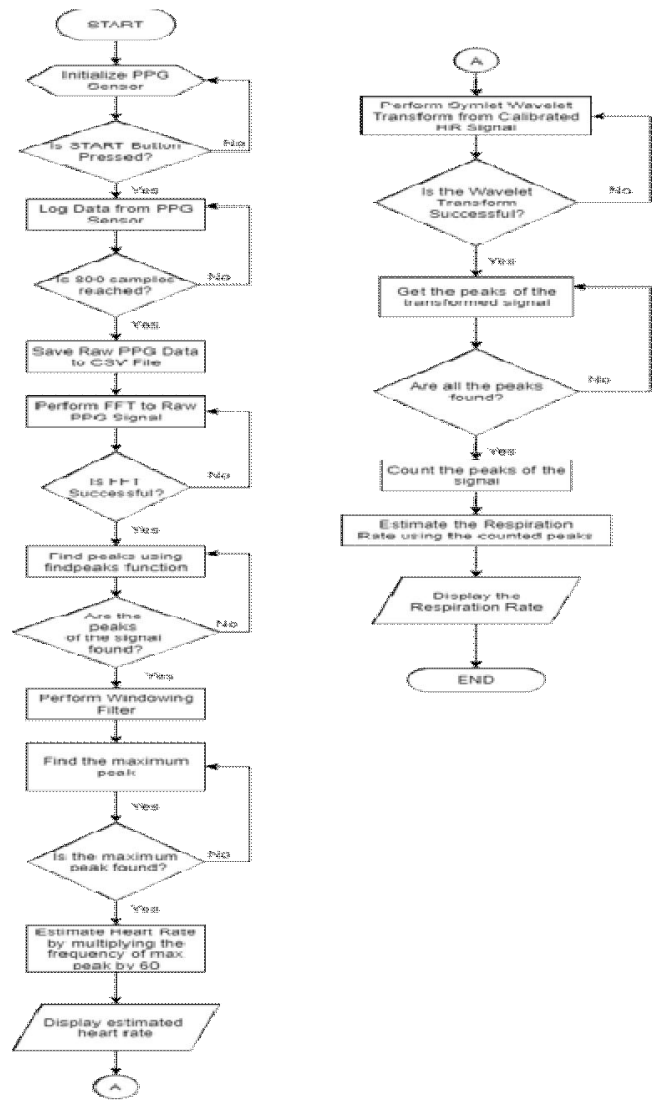

Figure 5: Complete System Flowchart
Figure 5 shows the complete system flowchart of this study. To start, the PPG sensor must be initialized by placing the finger of the respondent in the custom pulse oximeter. The start button must be pressed to start the assessment. When the 800 samples are reached during the assessment, it will automatically save as a CSV file. Matlab will then perform the Fast Fourier Transform to filter, window, and estimate the heart rate. Since the respiratory signal is carried in the heart rate signal, Matlab will perform the Symlet Wavelet Transform to estimate the respiratory rate.

\subsection{Heart Rate Acquisition}

The process of preparing the heart rate sample are as follows: (1) place the respondent's finger in the custom pulse oximeter, (2) The custom pulse oximeter will be connected to the microcontroller analog to digital converter, (3) Connect the microcontroller analog to digital converter to the computer, (4) Open the Matlab software then run the program, (5) The graphical user interface will appear then the patient's name should be the input, (6) Press the start button for assessment, (6) PPG will be plotted using Matlab, (7) The heart rate is the frequency times 60 bits per minute (8) The user will see the output through the graphical user interface.

\subsection{Fast Fourier Transform for Filtering, Windowing, and Estimating Heart Rate}

The Fast Fourier Transform is the algorithm used to filter, window, and estimate the Heart Rate. As stated by O'Haver, $\mathrm{T}$., the Fourier Filter is a type of filtering function based on manipulating specific frequency components of a signal. The Fourier Transform of the signal is then taken, leading to attenuation or amplification of a particular frequency and inverting the transform [8]. Fast Fourier Transform is used since it is the most basic algorithm for filtering, windowing, and extracting. It can measure a lot of the input, and the signal would still be there. Even though it has a small window, it will not distort the signal. Equation 1 shows the formula and syntax for Fast Fourier Transform.

$$
\begin{aligned}
& \mathrm{F}(\mathrm{x})=\sum_{n=0}^{N-1} f(n) e^{-j 2 \pi\left(\frac{n}{N}\right)} \\
& \mathrm{f}(\mathrm{n})=\frac{1}{N} \sum_{n=0}^{N-1} F(x) e^{j 2 \pi\left(x \frac{n}{N}\right)}
\end{aligned}
$$

Wherein:

$\mathrm{F}(\mathrm{x})=$ sample in frequency

$\mathrm{F}(\mathrm{n})=$ sample in time

$\mathrm{n}=$ time index

$\mathrm{X}=$ frequency index

$\mathrm{N}=$ number of samples

In this study, during the assessment, MatLab will count from 0 to 800 since there will be 800 samples and also the corresponding y values which is the ADC value. Per count, corresponding data points will be considered as the input or 
$\mathrm{f}(\mathrm{n})$. The frequency index is from $0 \mathrm{~Hz}$ to $7 \mathrm{~Hz}$. $\mathrm{F}(\mathrm{x})$ will be the output which is already the signal wherein the Fast Fourier Transform was used. Lastly, the number of samples or $\mathrm{N}$ is equivalent to 800 samples, as stated earlier.

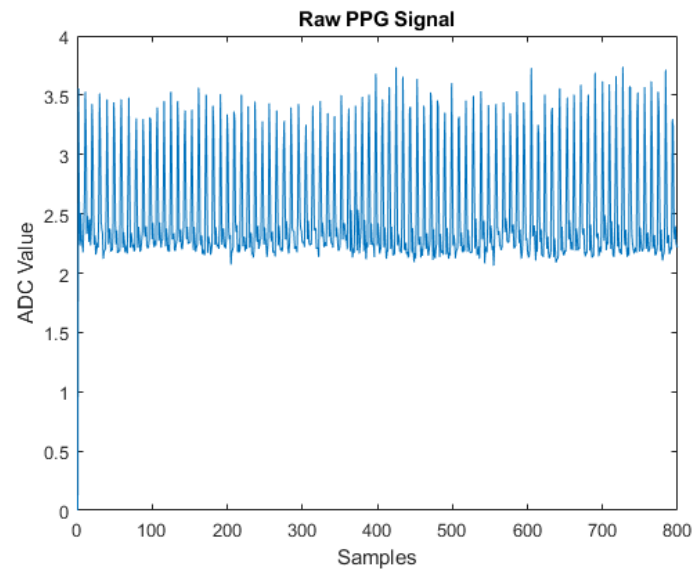

Figure 6: Sample Raw PPG Signal

The first step is to get the data from Arduino, as seen in Figure 6. In 1 minute, there will be 800 samples. Next is to use the Fast Fourier Transform. The Fast Fourier Transform is an algorithm that converts a time-domain signal into the frequency domain. We determine the frequency of the signal produced by the pulse sensor at a given time. Figure 7 shows the power spectral density plot upon using the Fast Fourier Transform. After using the Fast Fourier Transform, find the signal peaks using MatLab's find peaks in the frequency domain spectrum. After identifying the peak, the next step is to use a bandpass filter. Take note that the frequency must be $0.5 \mathrm{~Hz}$ to $2 \mathrm{~Hz}$, wherein the beats per minute will be 30 beats per minute to 120 beats per minute. The highest peak frequency is multiplied by 60 to calculate the heart rate in beats per minute.

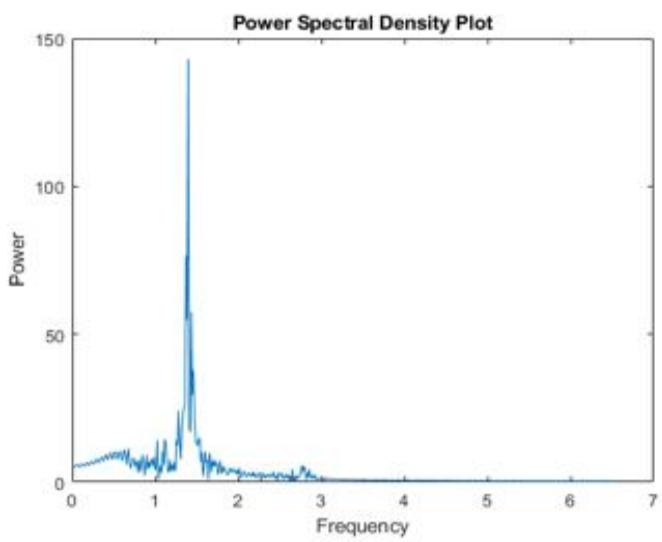

Figure 7: Sample Signal After Using Fast Fourier Transform

\subsection{Wavelet Transform for Extracting Respiratory Rate}

The Symlet Wavelet Transform is the algorithm used to extract the Respiratory Rate from the photoplethysmography data. The Symlet Wavelet Transform is the most promising than any other Digital Signal Processing Algorithm and can provide easily interpretable visual representation signals. This algorithm specializes in applications such as selective modifications of signals or pattern recognition. According to the study made by B. Viyajkumari, J. Ganga Devi, and M. IndhuMathi, the Symlet Wavelet Transform is a better version of the Daubechies Wavelet Transform. This is because it yields a greater denoising feature to signal. Also, using the Symlet Wavelet Transform resembles the most QRS Complex of the PPG signal as compared to any other Wavelet Transform [9]. Equation 2 shows the Wavelet Transform equation [10].

$$
F(a, b)=\int_{-\infty}^{\infty} f(x) \psi_{(a, b)}^{*}(x) \mathrm{d} x
$$

Wherein:

$\psi^{*}(\mathrm{a}, \mathrm{b})=$ wavelet analyzing function

$\mathrm{F}(\mathrm{x})=$ function of time

In this study, $f(x)$ or the function of time is the photoplethsymogram (PPG) raw signal. The wavelet analyzing function will capture the low frequencies and will define the location of the wavelet. $F(a, b)$ is the output which is the signal wherein the Wavelet Transform was used.

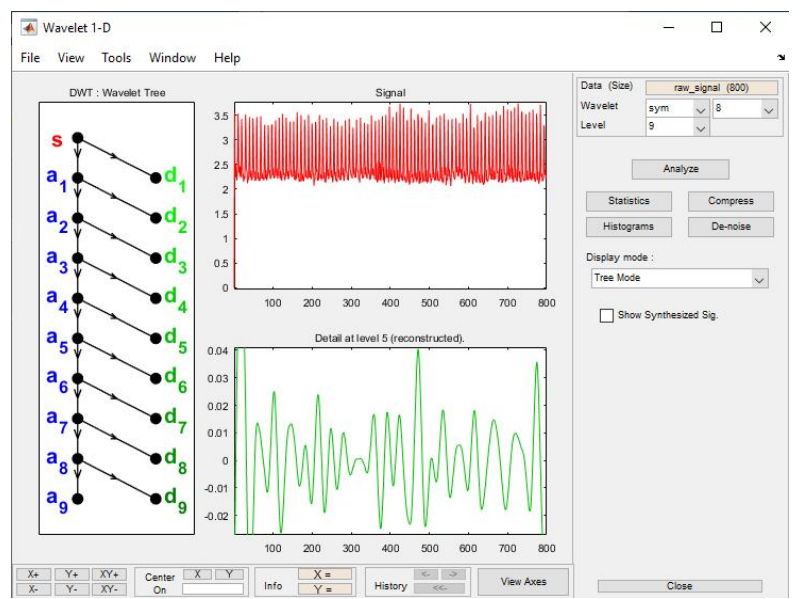

Figure 8: Application of Symlet Wavelet Transform

To extract and calculate the respiratory rate, the Symlet Wavelet Transform is used, as seen in Figure 8. Next, find the peaks with a minimum of 0.001 . After which is the calculation of the approximate respiratory rate, as seen in Figure 9.

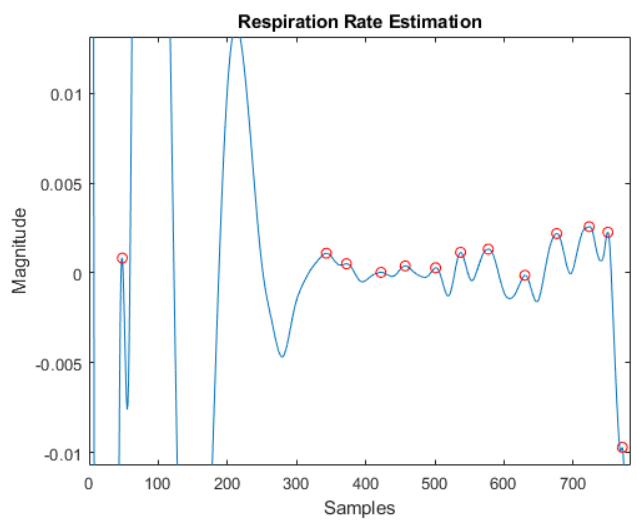

Figure 9: Estimation of Respiratory Rate 


\subsection{Output Accuracy Test}

Mean Squared Error compares the performance of both device's data for validation. For verification, the data gathered from the custom pulse oximeter will correspond to the clinically graded one. Mean Squared Error is then used to determine how close the data collected (Heart Rate and Respiratory Rate) from both devices. Mean Squared Error is the most common way of evaluation. It measures the average squared error of the predictions and calculates the square difference between the projections and the target and averages. The higher the value, the worse the model [11].

The Pulse Rate (PR) Accuracy Range is from 25-240 beats per minute from the clinical-grade pulse oximeter specification sheet, whereas the Respiration Rate (RRp) Accuracy Range is from 4-70 respiration per minute. The accuracy of the custom pulse oximeter is computed by using Mean Squared Error. The MatLab syntax to compute for the Mean Squared Error is:

Wherein:

$$
\text { Err=immse }(\mathrm{x}, \mathrm{y})
$$

$\mathrm{X}=$ values of the custom pulse oximeter

$\mathrm{Y}=$ values of the clinical pulse oximeter

Table 1: Complete Table of the Averaged Heart Ratefor Both Custom and Clinical Grade Pulse Oximeter

\begin{tabular}{|c|c|c|c|}
\hline \multicolumn{5}{|c|}{ Heart Rate } \\
\hline Sample No. & $\begin{array}{c}\text { Clinical } \\
\text { Grade Pulse } \\
\text { Oximeter }\end{array}$ & $\begin{array}{c}\text { Custom } \\
\text { Pulse } \\
\text { Oximeter }\end{array}$ & $\begin{array}{c}\text { Percent } \\
\text { Difference }\end{array}$ \\
\hline 1 & 92.67 & 92.33 & 0.368 \\
\hline 2 & 88.67 & 88.33 & 0.384 \\
\hline 3 & 74 & 73.33 & 0.910 \\
\hline 4 & 88.67 & 88 & 0.758 \\
\hline 5 & 78 & 79.33 & 1.691 \\
\hline 6 & 89.33 & 87.67 & 1.876 \\
\hline 7 & 90.33 & 90 & 0.366 \\
\hline 8 & 82.33 & 82.33 & 0 \\
\hline 9 & 66.67 & 67.44 & 1.148 \\
\hline 10 & 83.67 & 86.33 & 3.129 \\
\hline 11 & 86.33 & 85.33 & 1.165 \\
\hline 12 & 86.33 & 86.67 & 0.393 \\
\hline 13 & 81.67 & 81.67 & 0 \\
\hline 14 & 77 & 79.33 & 2.981 \\
\hline 15 & 108 & 106.33 & 1.558 \\
\hline 16 & 103 & 103.67 & 0.648 \\
\hline 17 & 88.67 & 88 & 0.758 \\
\hline 18 & 105.67 & 106.33 & 0.623 \\
\hline 19 & 99.33 & 97.67 & 1.685 \\
\hline 20 & 71.67 & 71.67 & 0 \\
\hline 21 & 72.33 & 70 & 3.274 \\
\hline 22 & 101.67 & 102 & 0.324 \\
\hline 23 & 66.33 & 66.67 & 0.511 \\
\hline 24 & 75.33 & 74.67 & 0.88 \\
\hline 25 & 71.67 & 71.33 & 0.476 \\
\hline 26 & 65.67 & 67 & 2.005 \\
\hline & & & \\
\hline
\end{tabular}

\begin{tabular}{|c|c|c|c|}
\hline 27 & 75.33 & 76 & 0.885 \\
\hline 28 & 75.67 & 73.67 & 2.678 \\
\hline 29 & 104 & 105.33 & 1.271 \\
\hline 30 & 85.67 & 86 & 0.384 \\
\hline
\end{tabular}

Table 1 shows the averaged heart rate from the custom pulse oximeter alongside the measurement of the number of large squares in one R-R interval from the clinical grade pulse oximeter based on three trials and the percent difference. All 30 samples show a heart rate within the normal range based on the gathered data. As for the percent difference, there is just a small difference between the results of the custom pulse oximeter and the clinical grade pulse oximeter.

Table 2: Complete Table of the Averaged Respiratory Rate for Both Custom and Clinical Grade Pulse Oximeter

\begin{tabular}{|c|c|c|c|}
\hline \multicolumn{4}{|c|}{ Respiratory Rate } \\
\hline Sample No. & $\begin{array}{c}\text { Clinical } \\
\text { Grade Pulse } \\
\text { Oximeter }\end{array}$ & $\begin{array}{l}\text { Custom } \\
\text { Pulse } \\
\text { Oximeter }\end{array}$ & $\begin{array}{c}\text { Percent } \\
\text { Difference }\end{array}$ \\
\hline 1 & 11.33 & 11.67 & 2.957 \\
\hline 2 & 19 & 19 & 0 \\
\hline 3 & 15.33 & 14.67 & 4.4 \\
\hline 4 & 12.67 & 13 & 2.571 \\
\hline 5 & 13.67 & 11.33 & 18.72 \\
\hline 6 & 17.67 & 18.67 & 5.504 \\
\hline 7 & 16.67 & 16.33 & 2.061 \\
\hline 8 & 16 & 15 & 6.452 \\
\hline 9 & 13.67 & 11.33 & 18.72 \\
\hline 10 & 13 & 11.33 & 13.728 \\
\hline 11 & 20 & 17.33 & 14.305 \\
\hline 12 & 23.67 & 23.67 & 0 \\
\hline 13 & 17 & 16 & 6.061 \\
\hline 14 & 17.33 & 14.33 & 18.952 \\
\hline 15 & 15.67 & 13.33 & 16.138 \\
\hline 16 & 23 & 23 & 0 \\
\hline 17 & 12 & 11 & 8.696 \\
\hline 18 & 13.33 & 10 & 28.547 \\
\hline 19 & 14.67 & 12.33 & 17.333 \\
\hline 20 & 13.67 & 12 & 13.011 \\
\hline 21 & 12 & 11.33 & 5.744 \\
\hline 22 & 13 & 10.67 & 19.687 \\
\hline 23 & 13 & 11.67 & 10.782 \\
\hline 24 & 12.67 & 11 & 14.111 \\
\hline 25 & 12.33 & 11.33 & 8.453 \\
\hline 26 & 12.67 & 11 & 14.111 \\
\hline 27 & 12.67 & 12 & 5.432 \\
\hline 28 & 14 & 11.67 & 18.154 \\
\hline 29 & 12.33 & 10.33 & 17.652 \\
\hline 30 & 16.67 & 16.67 & 0 \\
\hline
\end{tabular}

Table 2 shows the measured averaged respiratory rate from the custom pulse oximeter from all 30 samples and all three trials alongside the measurement from the reference clinical grade pulse oximeter. It can be seen that the gathered data is in the normal range. The last column also shows the calculated percent difference to compare both outputs. 


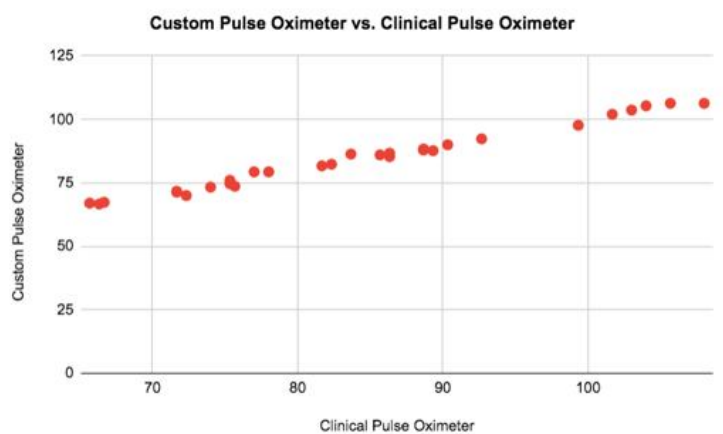

Figure 10: Graph of the Heart Rate Acquired from Both Custom Pulse Oximeter and Clinical Pulse Oximeter

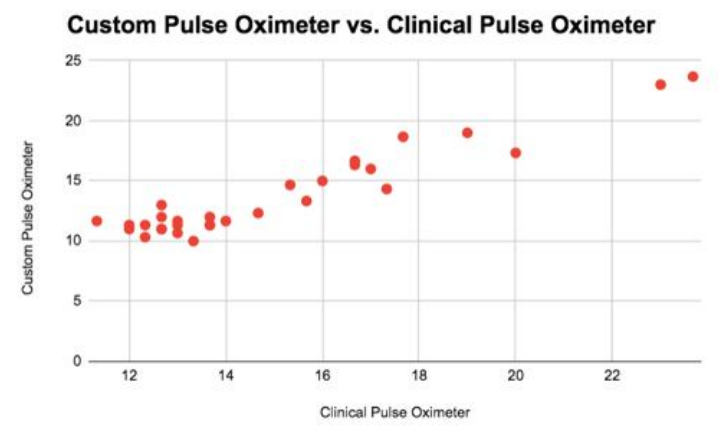

Figure 11: Graph of the Respiratory Rate Acquired from Both Custom Pulse Oximeter and Clinical Pulse Oximeter

Figure 10 shows the graph comparing the averaged heart rate. On the other hand, Figure 11 shows the graph comparing the averaged respiratory rate acquired from all 30 samples and all three trials of the custom pulse oximeter and clinical-grade pulse oximeter. It shows almost a linear graph which can be concluded that there is a small difference between the values garnered from both clinical and custom pulse oximeter.

The Mean Squared Error of the total Heart Rate and total Respiration Rate was computed by usingMatLab. The Mean Squared Error for the Heart Rate for the first trial is 3.6, the Mean Squared Error for the Respiratory Rate for the first trial is 4.33. For the second trial, the Mean Squared Error for the Heart Rate and Respiratory Rate is 5.5 and 3.3333, respectively. Lastly, for the third trial, the Mean Squared Error for the Heart Rate and Respiratory Rate is 2.3 and 3.63, respectively. The total Mean Squared Error for the Heart Rate is 3.8 and 3.7667 for the Respiratory Rate, as seen in Figures 11 and 12 . With the presented data, it can be said that the error is low because the Mean Squared Error shows a low value.

\section{CONCLUSION AND RECOMMENDATION}

Digital signal processing enabled the estimation of resting HR from PPG raw data. This was performed using FFT through proper filtering and windowing. MatLab is a helpful tool in employing this process.
Moreover, in extracting RR from HR, the Symlet Wavelet Transform was found to be the appropriate algorithm.

The validation of the performance of the proposed custom pulse oximeter against a reference pulse oximeter (clinical) was successfully performed with 30 multiple samples. All 30 samples, including all trials from both custom and reference pulse oximeter, resulted in a Mean Square Error (MSE) of 3.8 for $H R$ and 3.7667 for RR. The custom pulse oximeter performed $\mathrm{HR}$ and $\mathrm{RR}$ derivation, and the results are comparable to a clinical-grade device.

Future works can explore other algorithms considering other deriving RR from HR and consider other vital signs incorporated in the system. This can be beneficial in terms of portability for point of care applications.

\section{REFERENCES}

1. C. Kelly. Respiration rate 1: why accurate measurement and recording are crucial, Nursing Times, Vol. 114, pp. 23-24, April2018.

2. I. Wheatley. Respiratory rate 3: how to take an accurate measurement,Nursing Times, Vol. 114, pp. 21-22,June 2018.

3. T. O'Haver.A pragmatic introduction to signal processing,in Proc. of XXIV Conference of the Italian Association of Theoretical and Applied Mechanics (AIMETA), October 2019.

4. J.M. Kim, J.H. Hong, N.J. Kim, E. J. Cha and T.S. Lee. Two algorithms for detecting respiratory rate from ECG signal, in IFMBE Proceedings, Vol 14, Springer, Berlin, Heidelberg, 2008, pp. 1-3.

5. F. Weber-Muller, S. Reichert-Penetrat, J.L. Schmutz and A. Barbaud. Contact dermatitis from polyacrylate in TENS electrode, Annales de dermatologie et de vénéréologie, Vol. 131, pp. 478-480, June 2004.

6. N. Leelavathi, A. Abdul Rahman, B.K. Srikanth, S. Kalki Kumar. Signal Processing Electrocardiogram using Wavelet Transform Based on Mallet Fast Algorithm, International Journal of Advanced Trends in Computer Science and Engineering, Vol. 7, pp. 127-131, Nov 2018.

7. C.O.Manlises, J.C.Dela Cruz, L.M.A.Muralla, D.M.T.Payas, andM.J.T.Posada. Monitoring of Blood Pressure Using Photoplethysmography (PPG) Sensor with Aromatherapy Diffusion, $20166^{\text {th }}$ IEEE International Conference on Control System, Computing and Engineering (ICCSCE), pp. 476-480, Nov 2016.

8. L. Zhao, S. Reisman and T. Findley, Derivation of respiration from electrocardiogram during heart rate variability studies, Computers in Cardiology 1994, Bethesda, MD, USA, 1994, pp. 53-56.

9. B. Vijayakumari, J. G. Devi and M. I. Mathi, Analysis of noise removal in ECG signal using symlet wavelet,2016 International Conference on Computing Technologies and Intelligent Data Engineering (ICCTIDE'16), pp. 1-6, Jan 2016. 
10. S. Faziludeen and P.V. Sabiq. ECG beat classification using wavelets and SVM. 2013 IEEE conference on Information and Communication Technologies, 2013, pp. 815-818.

11. A.K. Yadav, A.P. Kumar and S. Kumar.De-noising of ultrasound image using discrete wavelet transform by symlet wavelet and filters, 2015 International Conference on Advances in Computing, Communications and Informatics (ICACCI), 2015, pp. 1204-1208. 